\title{
Modalités et fonctions de l'éloge dans les discours des présidents américains contemporains
}

\author{
Luc Benoit à la Guillaume
}

\section{(2) OpenEdition \\ 1 Journals}

\section{Édition électronique}

URL : http://journals.openedition.org/rhetorique/662

DOI : $10.4000 /$ rhetorique.662

ISSN : 2270-6909

\section{Éditeur}

UGA Éditions/Université Grenoble Alpes

\section{Édition imprimée}

ISBN : 978-2-37747-062-4

\section{Référence électronique}

Luc Benoit à la Guillaume, « Modalités et fonctions de l'éloge dans les discours des présidents américains contemporains », Exercices de rhétorique [En ligne], 11 | 2018, mis en ligne le 05 octobre 2018, consulté le 12 septembre 2020. URL : http://journals.openedition.org/rhetorique/662 ; DOI : https://doi.org/10.4000/rhetorique.662

Ce document a été généré automatiquement le 12 septembre 2020.

\section{(c) (P)}

Les contenus de la revue Exercices de rhétorique sont mis à disposition selon les termes de la Licence Creative Commons Attribution - Pas d'Utilisation Commerciale - Partage dans les Mêmes Conditions 4.0 International. 


\title{
Modalités et fonctions de l'éloge dans les discours des présidents américains contemporains
}

\author{
Luc Benoit à la Guillaume
}

1 Les présidents américains prononcent des éloges publics afin de rappeler les valeurs fondatrices de la nation américaine. Ainsi les discours d'investiture, genre de discours qui remonte aux origines de la République étatsunienne, célèbrent la stabilité des institutions américaines et le transfert de pouvoir entre présidents successifs tout en rappelant les valeurs qui unissent la nation. Quant au discours de Gettysburg d'Abraham Lincoln, largement inspiré des oraisons funèbres de Périclès, il rend hommage aux morts de cette bataille sanglante pour mieux redéfinir le sens d'une nation dont l'existence même était l'enjeu de la guerre de Sécession' ${ }^{1}$. Les modalités cérémonielles et la fonction consensuelle de l'éloge présidentiel semblent donc, au premier abord, parfaitement clairs et paraissent relever en tous points du genre épidictique, dont Laurent Pernot nous rappelle que, depuis l'Antiquité, sa «fonction principale est sociale » et qu'elle « donne forme aux représentations et aux croyances partagées par le groupe, explicite et justifie des valeurs communément admises et en propose parfois de nouvelles ${ }^{2}$. " Traditionnellement, l'éloge permet au chef de l'État de rappeler les valeurs américaines lors de grandes cérémonies officielles, qu'il s'agisse de rendre hommage aux morts, de commémorer des événements historiques majeurs ou plus généralement de marquer les moments importants dans la vie de la nation. Toutefois, la récente prolifération de discours cérémoniels vient nous suggérer que le genre épidictique n'a pas pour seule fonction de rappeler dans un cadre cérémoniel les valeurs communes et nous incite à reposer la question des modalités et de la fonction de l'éloge. Cette question, à vrai dire, n'a jamais été évidente, et ce dès l'origine du genre épidictique, «concept à la dérive » qui s'applique à la fois aux « discours d'éloge et de blâme » au sens strict et, dans un sens plus large, à « l'éloquence d'apparat » sous toutes ses formes ${ }^{3}$. Quittant le cérémonial empesé des grandes occasions officielles, il s'insinue là où on ne l'attend pas, notamment dans les discours sur l'état de l'Union, qui relèvent du genre délibératif et contiennent cependant de plus en plus d'éloges de 
héros anonymes. Il loue des communautés particulières lors d'innombrables cérémonies d'importance mineure, des héros peu connus lors du traditionnel discours sur l'état de l'Union, des étudiants parfois médiocres pendant les cérémonies de remise de diplômes (commencement), voire des dindes, qui bénéficient du droit de grâce lors de la fête traditionnelle de Thanksgiving. Il flirte avec l'humour et le paradoxe et retrouve le caractère spectaculaire de happening qui est le sien depuis ses origines sophistiques ${ }^{4}$. L'éloge des présidents américains est donc de plus en plus diversifié, opportuniste et spectaculaire. Afin de mieux cerner les modalités et de comprendre les fonctions des éloges présidentiels contemporains, on commencera par en étudier la diversité croissante, avant d'analyser les stratégies opportunistes dont ils font l'objet, kairos oblige. Enfin on montrera que l'éloge présidentiel tient de plus en plus du happening, pour des raisons qui sont liées à la médiatisation de la vie politique contemporaine.

\section{Dire la valeur d'une nation en s'adressant à chacune de ses composantes}

2 Au vingtième siècle, l'avènement d'une présidence rhétorique ${ }^{5} \mathrm{a}$ conduit les présidents à multiplier les prises de parole au-delà des grandes cérémonies officielles afin de renforcer le rôle du pouvoir exécutif face au Congrès. La décision du président Wilson de renouer en 1913 avec la tradition abandonnée un siècle plus tôt de prononcer en personne le discours sur l'état de l'Union devant le Congrès était emblématique de cette volonté du président de ne plus se contenter d'un rôle de maître des cérémonies officielles. Il s'ensuivit une augmentation et une diversification spectaculaire des prises de parole des présidents lors des campagnes électorales, face aux médias ou devant le Congrès ${ }^{6}$. Si la présidentialisation du régime a donné lieu à des discours relevant du genre délibératif, comme les discours sur l'état de l'Union ou les discours de campagne électorale, elle n'a pas pour autant conduit les présidents à négliger le genre épidictique et les éloges officiels. C'est même au vingtième siècle que les présidents se sont mis pleinement à jouer le rôle de grands prêtres de la religion civile, notamment lors des cérémonies d'investiture, comme l'a montré Robert Bellah dans un article célèbre qui prend pour exemple le discours d'investiture de John F. Kennedy en 1961. Bellah remarque que les références explicites à un Dieu qui protège l'Amérique se trouvent dans les discours épidictiques comme les discours d'investiture et non dans les discours délibératifs prononcés devant le Congrès et que le Dieu de ces discours reste vague, afin de convenir aux différentes traditions religieuses qui cohabitent aux États-Unis ${ }^{7}$. Les présidents participent donc au rappel des valeurs qui unissent l'Amérique lors d'un «calendrier rituel annuel » de la religion civile qui inclut la fête nationale le 4 juillet, la journée d'hommage aux morts (Memorial Day) et aux anciens combattants (Veterans' Day). Les valeurs patriotiques que ces discours rappellent reçoivent un supplément de légitimité religieuse, renforçant le caractère exceptionnel de la nation américaine.

3 La célébration des valeurs de l'Amérique reflète la domination d'un nationalisme culturel qui a émergé pendant la première moitié du vingtième siècle afin de répondre à « une série de crises, la guerre, le conflit de classe et la crise économique. Depuis les années 1960 au contraire, le pouvoir de l'État-nation a été plus largement contesté et les expressions publiques des mémoires locales se sont développées ${ }^{8} »$. Dans The Sound of Leadership, paru en 1987, Roderick Hart remarque une nouvelle augmentation du 
nombre de discours prononcés par les présidents américains et un changement de nature de la parole présidentielle, qui fait une place toujours plus grande aux prises de parole cérémonielles depuis les années 1960 :

C'est ici qu'il est important de remarquer que les présidents des années 1960 (en gros, de Kennedy à Nixon) et les présidents plus récents (de Ford à Reagan) ont participé à des événements à caractère cérémonial deux fois plus souvent en proportion, c'est-à-dire quatre fois plus souvent en chiffres bruts, que les présidents des années 1930 à 1950 . Ces cérémonies ont proliféré aussi bien à Washington que dans les États ; aux États-Unis comme à l'étranger. Depuis Ford, les présidents ont fait du moindre événement une cérémonie grandiose (par exemple, la remise du trophée lors des championnats de football américain); ils ont commémoré tous les héros de guerre, tous les bâtiments universitaires et tous les événements historiques disponibles; et ils ont introduit des discours pour célébrer des événements qui n'auraient sans doute pas sinon été considérés comme digne d'être célébrés (par exemple les discours au départ et à l'arrivée des voyages en avion) ainsi que lors de cérémonies auparavant dénuées de morceaux de bravoure rhétoriques (par exemple lors de la signature des lois ${ }^{9}$ ).

4 Une telle évolution pose dès cette époque la question des modalités et de la fonction de l'éloge présidentiel, dans la mesure où l'augmentation du nombre d'allocutions s'accompagne d'un changement de lieux (ailleurs qu'à Washington) et de nature (cérémonies mineures). Dès lors, la question de la fonction de ces éloges se pose elle aussi à nouveaux frais car une telle prolifération de discours peut difficilement servir uniquement au rappel des valeurs de la nation. Ces milliers de discours cérémoniaux mineurs s'adressent le plus souvent non pas à la nation toute entière mais à l'une de ses composantes, professionnelle, générationnelle, ethnique ou religieuse. Ils témoignent de la fragmentation de la mémoire nationale, qui n'est plus exclusivement contrôlée par l'État fédéral et se décentralise de plus en plus. Elle donne lieu à ce que les speechwriters appellent le Rose garden rubbish. John C. Humes donne une définition de cette expression :

Pour chaque discours d'investiture il y a des milliers de discours cérémoniaux. Peter Benchley a rédigé les discours de Lyndon Johnson (jusqu'en 1968, où il s'est mis à écrire Les Dents de la mer). Benchley a donné un nom à ces discours informels «L'inauguration des chrysanthèmes » (Rose garden rubbish). C'est ainsi que Benchley décrivait le cocktail de louanges, de félicitations et de salutations que prononce le président lorsqu'il passe du bureau ovale au Rose garden afin de saluer les représentants d'organisations caritatives ou d'allumer l'arbre de Noël national en adressant ses meilleurs vœux. [...] Pourtant il ne faut pas sous-estimer les discours cérémoniaux ${ }^{10}$.

5 La prolifération de discours cérémoniaux mineurs n'est pas facile à interpréter. Il est tentant de la relier au contexte politique des années 1970 et 80 et d'y voir une réponse idéologique à la crise multiforme du système politique américain après la guerre du Viêtnam et le scandale du Watergate. Ce fut notamment le cas du président Ford lors des cérémonies du Bicentenaire de la Révolution américaine en juillet 1976, quelques mois avant l'élection présidentielle. Mais la confirmation de cette tendance depuis lors rend l'explication conjoncturelle insuffisante. En effet, la période récente a confirmé les évolutions étudiées par Hart. Depuis les années 1990, les présidents américains ont multiplié les cérémonies mineures à la Maison-Blanche et en dehors. Ils ont systématiquement pris la parole alors qu'ils ne le faisaient pas auparavant, comme le montrent entre autres l'éloge des anciens combattants et des soldats morts pour la patrie. Tandis que le premier, Veterans' Day, est apparu après la Première guerre mondiale et est célébré le 11 novembre, le second, Memorial Day, date de la Guerre de 
Sécession et a lieu à la fin du mois de mai. Ces deux hommages existaient depuis de nombreuses décennies lorsque les présidents ont décidé, à partir des années 1990, de systématiquement prendre la parole pour marquer ces évènements. Lors de la journée du souvenir, la plupart des discours sont prononcés au cimetière national d'Arlington et donnent lieu à l'éloge de soldats ordinaires. Les présidents reprennent à cette occasion une pratique inventée par le président Reagan lors de ses discours sur l'état de l'Union et reprise par tous ses successeurs ${ }^{11}$. Il apparaît donc bien que les présidents prononcent des éloges dans des circonstances de plus en plus variées. Ils pratiquent l'éloge chaque fois qu'ils l'estiment nécessaire et qu'ils en tirent un avantage politique. C'est donc l'opportunité, voire l'opportunisme, des éloges présidentiels qu'il faut maintenant examiner.

\section{L'éloge à chaque fois que c'est possible et/ou nécessaire}

6 L'éloge présidentiel américain répond aux occasions qui se présentent, kairos oblige, qu'il s'agisse de répondre à une crise soudaine ou d'utiliser une cérémonie ordinaire à des fins politiques, voire d'injecter de l'éloge et du cérémonial dans des occasions qui en sont d'ordinaire dépourvues. Ce faisant, l'éloge présidentiel américain contemporain renoue avec ses racines sophistiques, telles que les décrit Barbara Cassin : il permet de dire « la valeur [...] chaque fois que l'occasion (kairos) s'en présente ${ }^{12} »$. Encore faut-il distinguer deux types d'occasions : celles que le président se doit d'utiliser et celles dont il se saisit opportunément. La première catégorie renvoie notamment aux discours de crise, que les présidents sont sommés de faire sauf à abdiquer le rôle qui est le leur depuis la réponse que Franklin D. Roosevelt a apportée à la crise de 1929. Du premier discours d'investiture de Roosevelt en mars 1933 jusqu'au discours du président Obama en 2012 lors de l'ouragan Sandy ou suite au meurtre de neuf Noirs, les présidents jouent le rôle d'« aumôniers des victimes ${ }^{13}$ ». S'ils omettent d'intervenir ou le font de manière inconsidérée, comme ce fut le cas pour George W. Bush après l'ouragan Katrina à la Nouvelle Orléans et, tout récemment, pour le président Trump suite au meurtre raciste d'une militante antifasciste à Charlottesville, ils payent un prix politique élevé. Le second type d'éloge renvoie à l'utilisation opportuniste et multiforme de l'éloge dans des contextes parfois inattendus, notamment lors des discours sur l'état de l'Union. La distinction entre les deux types d'occasions n'est pas étanche : les discours de crise donnent aussi lieu à une instrumentalisation politique.

7 Le discours présidentiel de crise est inséparable de la présidentialisation du régime politique américain depuis la présidence de Franklin D. Roosevelt. Le premier discours d'investiture de mars 1933 est tout à fait exemplaire à cet égard. Prononcé au pire moment de la grande dépression, alors que 25 \% des Américains étaient au chômage et que le système bancaire était en train de s'effondrer, il permit au nouveau président de rappeler en termes bibliques les valeurs de la nation en les opposant à celles des spéculateurs (les "money changers", véritables marchands qui avaient trahi le « temple » de la civilisation) et de réclamer les pleins pouvoirs face au Congrès au nom de l'urgence de la guerre économique et du mandat que le peuple lui avait confié. Depuis Roosevelt, tous les présidents interviennent publiquement en cas de crise, rappellent les valeurs qui unissent la nation et montrent la voie au Congrès. Il en fut ainsi au lendemain de Pearl Harbor en décembre 1941, que Roosevelt qualifia de 
manière célèbre de "day that will live in infamy " jusqu'aux suites du 11 septembre 2001, en passant par les moments-clés de la lutte pour les droits civiques pendant les années 1960 et les accidents ou les catastrophes tels que l'explosion de la navette Challenger en 1986 ou l'ouragan Sandy en 2012. Même lorsqu'ils blâment un ennemi, comme ce fut le cas après l'attaque japonaise de Pearl Harbor, ils n'en font pas moins l'éloge des valeurs américaines et c'est au nom de cette supériorité morale que le pays lutte. Il en va de même des interventions du président après les attentats islamistes du 11 septembre 2001. Le président Bush fit d'ailleurs un éloge de l'Islam dans une mosquée le 17 septembre 2001 pour prévenir toute réaction violente contre les Musulmans vivant aux États-Unis mais aussi pour mettre en avant les valeurs de tolérance et de liberté religieuse au nom desquelles les États-Unis mèneraient dorénavant leur guerre contre le terrorisme. Si tous les discours de crise ne sont pas exclusivement épidictiques, dans la mesure où ils proposent parfois des mesures d'urgence en réponse à la catastrophe, ils contiennent tous un élément de show épidictique et de rappel aux valeurs qui unissent l'Amérique ${ }^{14}$. Il arrive parfois que la crise tombe à point nommé et donne l'occasion au président de faire l'éloge de la nation à un moment particulièrement favorable sur le plan politique. L'ouragan Sandy frappa la côte est des États-Unis quelques jours avant l'élection présidentielle de 2012, qui s'annonçait serrée, et donna l'occasion à Barack Obama d'endosser le rôle de père de la nation et de rappeler le rôle de l'État fédéral lors de désastres de ce type, alors que son concurrent républicain Mitt Romney était partisan d'une réduction du rôle de la puissance publique. La ritualisation des crises fait partie des stratégies du président sortant qui cherche à se faire réélire ${ }^{15}$. L'avantage politique escompté conduit parfois les présidents à ritualiser des évènements marquants afin de tenter de faire perdurer le sentiment d'unité nationale. Ainsi, le choc créé dans l'opinion par les attentats du 11 septembre 2001 et le réflexe d'unité autour du président Bush a conduit ce dernier et son successeur Obama à instaurer un Patriot Day le 11 septembre. Cette journée donne lieu chaque année à des discours commémoratifs qui font l'éloge des valeurs de l'Amérique ${ }^{16}$.

Il arrive aussi que les présidents détournent un éloge convenu afin de faire passer un message qui l'est moins. Ce fut notamment le cas du président Bush lors de la cérémonie de remise des diplômes (commencement) à l'université Yale en 2001. Il est d'usage qu'une personne célèbre prononce un discours devant les étudiants et leurs parents à la fin de l'année universitaire. Bien souvent, les orateurs font l'éloge de l'institution universitaire et des valeurs de l'éducation, tout en profitant de l'occasion pour évoquer des sujets importants. En 2001, lors du tricentenaire de l'université Yale, le président nouvellement élu fut invité à prononcer un discours à l'université où il avait étudié à la fin des années 1960. Tout en faisant l'éloge de Yale et de ses professeurs que l'on attendait de lui dans la seconde partie de son allocution, le président Bush rappela avec insistance son parcours de cancre et son ancrage texan, loin des valeurs intellectuelles de la Nouvelle-Angleterre. Pour décrire son parcours peu orthodoxe et mettre les rieurs de son côté, il paraphrasa le poème célèbre de Robert Frost, The Road not Taken, qui décrit la conversion à la poésie, en parlant d'une " Academic Road less traveled ". Là où Robert Forst avait abandonné la Californie pour se consacrer à la poésie en Nouvelle-Angleterre, Bush avait fait le chemin inverse. Il prononça donc un éloge paradoxal de la paresse qui était une sorte de pied de nez aux élites liberal de la côte est, rappelant ainsi son ancrage dans les valeurs antiintellectuelles de l'Amérique profonde ${ }^{17}$. Quant à la pratique qui consiste à gracier des dindes lors de la fête de Thanksgiving, elle date de George H. W. Bush et a été suivie par 
ses successeurs. Elle s'apparente plutôt au pseudo-éloge, puisqu'il ne s'agit évidemment pas ici de faire passer un message sérieux de réhabilitation de valeurs oubliées mais de plaisanter devant les caméras ${ }^{18}$.

9 Enfin, il arrive que les présidents introduisent de l'éloge faute de pouvoir agir: ces discours consistent à dire au lieu de faire ou, de manière toute sophistique, à parler pour parler. Depuis la présidence de Ronald Reagan, les présidents américains se sont mis à faire l'éloge de héros ordinaires lors de leurs discours sur l'état de l'Union, introduisant une part d'éloge épidictique dans un discours fondamentalement délibératifi ${ }^{19}$. En 2016, le président Obama est allé encore plus loin en faisant de son discours une contemplation épidictique des valeurs de l'Amérique. Il a en effet prononcé son dernier discours sur l'état de l'Union devant les deux chambres du Congrès sans formuler la moindre proposition de loi, alors que, d'après l'article 2 alinéa 3 de la Constitution, le président «informera périodiquement le Congrès de l'état de l'Union, et recommandera à son attention les mesures qu'il estimera nécessaires et opportunes». Dans un contexte de polarisation croissante de la vie politique américaine et d'affaiblissement d'un président «lame duck » en fin de mandat, il s'est contenté de faire la liste des défis que le pays devrait affronter « les cinq prochaines années, les dix prochaines années ». Et, reprenant un thème que Ronald Reagan avait évoqué dans son premier discours d'investiture en 1981, il a conclu son allocution par un éloge des héros américains ordinaires en tous genres. Pour masquer son impuissance politique, le président Obama a donc été conduit à transformer un discours censé proposer un programme législatif en éloge de la nation, de ses valeurs et de ses citoyens.

Discours opportuniste, les éloges présidentiels s'adaptent aux circonstances et les exploitent, non sans éclat, tant leur «caractère spectaculaire» fait d'eux des « happenings » médiatiques ${ }^{20}$.

\section{Faire de l'éloge un spectacle}

11 En se généralisant, l'éloge présidentiel américain s'est diversifié et s'est adapté à de très nombreuses occasions, quitte à les exploiter à des fins politiques. Ce faisant, l'éloge s'est transformé en un spectacle adapté à l'ère électronique de la télévision et des nouveaux médias ${ }^{21}$. L'entreprise reaganienne de restauration des valeurs de l'Amérique après la crise des années 1970, marquées par la défaite au Viêtnam et le scandale du Watergate, se situait dans une conjoncture qui « exigeait que les dirigeants maîtrisent l'éloquence épidictique ${ }^{22} »$. Les talents d'ancien acteur de série B de Ronald Reagan le prédisposaient à la modernisation de la communication présidentielle. Les innovations qu'il a introduites ont parfois été reprises par ses successeurs. Reagan a commencé par moderniser les discours les plus traditionnels. Ainsi, dès son premier discours d'investiture prononcé le 20 janvier 1981, le nouveau président a adapté sa pratique de l'éloge à l'ère de la télévision, notamment dans la partie finale du discours, qui fut coproduite avec les grandes chaînes de télévision. Reagan y fait l'éloge des principaux monuments de la capitale américaine et les relie aux grands hommes, Washington, Jefferson et Lincoln, qui y sont associés. Le lieu même du discours a été déplacé du côté ouest du Capitole, qui donne directement sur ces monuments, et la magie de la télévision, avec ses gros plans, permet de projeter en direct l'image des monuments évoqués par le président. Ce clip vidéo patriotique se termine par une anecdote, celle 
de l'engagement patriotique du soldat Martin Treptow pendant la Première guerre mondiale, que Ronald Reagan exigea de conserver, en dépit de son inexactitude factuelle ${ }^{23}$.

Lors des cérémonies officielles qui font l'éloge des valeurs et des héros américains, Reagan a confirmé cette tendance au storytelling et au spectacle. Peggy Noonan, l'une de ses plumes les plus talentueuses, revendiquait d'ailleurs un rapport littéraire au discours politique qui la mettait parfois en porte-à-faux avec le Département d'État. Dans l'un de ses livres, elle cite l'exemple d'un discours prononcé lors d'un déplacement en Chine communiste qui contenait la métaphore "l'histoire est un fleuve ». Alors qu'elle défendait le caractère littéraire de cette image, le Département d'État voulait la censurer en accusant cette expression de valider une conception déterministe de l'histoire qui était incompatible avec la liberté ${ }^{24}$. Par ailleurs Noonan écrivit l'un des discours les plus réussis du président Reagan, lors de la commémoration du quarantième anniversaire du débarquement, à la Pointe du Hoc. Sur le lieu même des exploits des soldats américains, le président Reagan s'exclama : «Voici les gars de la pointe du Hoc. Voici les hommes qui ont pris les falaises. Voici les champions qui ont contribué à la libération d'un continent. Voici les héros qui ont contribué à terminer une guerre. » Le choix du lieu spectaculaire et le pathos, que renforçait l'anaphore, ont permis au vieil acteur de transformer cet éloge des soldats américains en séquence télévisuelle parfaite ${ }^{25}$.

13 Non contents d'adapter aux médias audiovisuels les grandes occasions cérémonielles, les présidents contemporains introduisent du spectacle dans des occasions qui en étaient dépourvues. Depuis les années 1960, les discours sont prononcés en début de soirée, afin de pouvoir être retransmis en direct à la télévision. Les discours sur l'état de l'Union de Ronald Reagan sont qualifiés de "succès épidictiques » mais aussi d'« échecs délibératifs » qui font passer le cérémonial avant le détail des propositions législatives ${ }^{26}$. En effet, Reagan dépoussière les discours sur l'état de l'Union en apportant un certain nombre d'innovations que tous ses successeurs ont reprises. Les discours sont désormais plus courts, afin de s'adapter au format télévisuel. Les présidents sont conduits à faire de ces discours des spectacles qui s'adressent au moins autant aux téléspectateurs qu'aux membres du Congrès.

14 C'est dans ce contexte que l'on peut comprendre le sens de l'éloge des héros ordinaires que le président Reagan a introduit dans ces discours. En 1982, il a invité Lenny Skutnik, qui avait plongé dans le fleuve Potomac en plein hiver afin de sauver la vie d'une victime d'un accident d'avion. Reagan a invité Skutnik et a raconté son histoire, déclenchant une standing ovation. Tous ses successeurs à la Maison-Blanche l'ont imité, si bien que le New York Times s'est mis à parler de syndrome Skutnik. « De 1982 à 2016, 35 discours sur l'état de l'Union ont été prononcés et les présidents ont cité 91 invités. $\mathrm{Au}$ total, sur ces 91 invités, 75, c'est-à-dire $82 \%$, étaient des héros ordinaires, des membres de la société civile cités en exemple ${ }^{27}$. » Lorsque les présidents font face à un Congrès contrôlé par l'opposition, ce qui n'est pas rare dans la période récente, l'éloge des héros ordinaires peut servir à défendre une politique de manière spectaculaire sans que cela mène à l'adoption de lois en rapport avec le principe défendu. En effet, depuis une vingtaine d'années, les rapports entre les deux principaux partis se sont considérablement dégradés. Les propositions formulées par le président lors de son discours sur l'état de l'Union ont assez peu de chances d'être adoptées par le Congrès. L'éloge spectaculaire d'un héros ordinaire dont l'histoire édifiante confirme les valeurs 
du président sert alors de substitut à l'action. Il faut à nouveau souligner le rôle pionnier de Ronald Reagan en la matière. Dès 1984, son éloge du sergent Trujillo lui permet de faire la promotion de l'invasion de la Grenade, qui avait été critiquée par le Congrès à majorité démocrate. En 1985, le président fait l'éloge de deux personnes, Jean Nguyen et Clara Hale, qui lui permettent de réhabiliter l'intervention américaine au Viêtnam et la politique des Républicains en direction des minorités noires. Les successeurs de Reagan utiliseront l'éloge du héros ordinaire devant le Congrès de manière similaire. Ici l'éloge présidentiel revient à parler pour se faire le porte-parole $\mathrm{du}$ peuple contre ses représentants, faute de pouvoir travailler de concert avec le Congrès pour faire voter des lois. L'éloge médiatique permet au président d'exercer une fonction tribunitienne ${ }^{28}$. Ces changements récents reflètent l'évolution des médias contemporains: la personnalisation, le spectaculaire, la fragmentation et la normalisation (personalization, dramatization, fragmentation, normalization ${ }^{29}$ ). Grâce à l'éloge d'un héros ordinaire, les présidents racontent des histoires exemplaires qui leur permettent de "réduire et de styliser, de concentrer les enjeux, de suggérer une exemplarité » directement diffusable sous forme d'extraits courts (soundbites) au journal télévisé du soir.

La pratique contemporaine de l'éloge par les présidents américains s'est considérablement diversifiée. Il ne s'agit plus seulement de rappeler les valeurs communes de la nation lors des grandes cérémonies officielles. Elle est désormais marquée par une très grande diversité qui pose la question du sens de cette pratique de plus en plus utilisée. La présence de l'éloge dans des discours délibératifs repose la question de l'extension de ce « concept à la dérive ». Ce qui semble prédominer, c'est la propension des présidents d'exploiter les occasions rhétoriques à des fins politiques et la tendance au spectacle. Il faut en permanence montrer que l'on gouverne, au détriment du gouvernement lui-même et au risque de l'impuissance ${ }^{30}$. Il y a sans doute des raisons spécifiquement américaines à cette situation. Dans un système qui concentre l'attention sur l'exécutif tout en donnant de solides contre-pouvoirs au Congrès et à la Cour Suprême et qui est marqué par un blocage institutionnel lié à la polarisation croissante entre les deux grands partis, le président a de plus en plus de mal à agir. Le recours systématique à l'éloge peut alors constituer un refuge. Il n'est toutefois pas l'apanage des Américains. Il faudrait se demander si cette évolution ne reflète pas aussi des évolutions plus générales, liées à la crise de la représentation politique, qui prend des formes différentes mais est perceptible dans nombre de nos démocraties.

\section{NOTES}

1. G. Wills, Lincoln at Gettysburg: The Words that Remade America, New York, Simon and Schuster, 1992.

2. L. Pernot, Epideictic Rhetoric. Questioning the Stakes of Ancient Praise, Austin, University of Texas Press, 2015, p. x. Sauf indication contraire, je suis l'auteur des traductions des citations. 
3. L. Pernot, La Rhétorique de l'Éloge dans le monde gréco-romain, Paris, Institut d'études augustiniennes, 1993, tome 1, p. 11 : «Que signifie, en effet, le mot "épidictique" ? Au sens strict, il recouvre la catégorie rhétorique des discours d'éloge et de blâme. Mais on l'applique aussi, dans un sens large, à tous les discours composés pour la montre (epideixis), c'est-à-dire aux diverses formes de l'éloquence d'apparat [...]. L'épidictique est un concept à la dérive, ce qui met en cause l'identité et la destination du genre. Lorsqu'on s'intéresse à l'éloge rhétorique, l'objet se présente au premier abord comme flou, gros d'ambiguïtés mal résolues. »

4. B. Cassin, L'Effet sophistique, Paris, Gallimard, 1995, p. 202.

5. Voir sur ce point Jeffrey Tulis, The Rhetorical Presidency, Princeton, Princeton University Press, 1987.

6. L. Benoit à la Guillaume, Quand la Maison-Blanche prend la parole : le discours présidentiel de Nixon à Obama, Berne, Peter Lang, 2012.

7. R. Bellah, «Civil Religion in America », dans Religion in America, ed. by William Mc Loughlin and Robert Bellah, Boston, Beacon Press, 1966.

8. J. Bodnar, Remaking America. Public Memory, Commemoration, and Patriotism in the Twentieth Century, Princeton, Princeton University Press, 1992, p. 251.

9. R. Hart, The Sound of Leadership: Presidential Communication in the Modern Age, Chicago, The University of Chicago Press, 1987, p. 17.

10. J.C.Humes, Confessions of a White House Ghost Writer: Five Presidents and Other Political Adventures, Washington DC, Regnery Publishing, 1997, p. 3.

11. L. Benoit à la Guillaume, «Stylistique de l'éloge du héros ordinaire dans les discours sur l'état de l'Union ", Études de stylistique anglaise, 10, Lyon, Université Jean Moulin Lyon 3, p. 15-28.

12. B. Cassin, op. cit., p. 200.

13. A. Lherm, «Bill Clinton, représentant de commerce d'une Amérique plurielle dans un monde global ? ", dans F. Durpaire et T. Snegarof éd., L'Unité réinventée. Les présidents américains face à la nation, Paris, Ellipses, 2008.

14. A. Kiewe éd., The Modern Presidency and Crisis Rhetoric, Westport, Praeger, 1994, p. xviii.

15. L. Benoit à la Guillaume, Quand la Maison-Blanche prend la parole, op. cit., p. 89-93.

16. Ibid., p. 92.

17. L. Benoit à la Guillaume, "Dualités rhétoriques : l'éloge paradoxal de George W. Bush à Yale », Bulletin de la société de stylistique anglaise, $\mathrm{n}^{\circ} 28$, Nanterre, Atelier intégré de reprographie, 2007, p. 217-228.

18. Pour la distinction entre différents types d'éloge je renvoie à Mathieu de la Gorce, « "Embabouiner" le lecteur: Détournements satiriques de la coopération fictionnelle dans le Tableau des differens de la religion, de Ph. de Marnix de Sainte-Aldegonde ", dans F. Lavocat dir., Usages et théories de la fiction. Le Débat contemporain à l'épreuve des textes anciens (XVIe-XVIIIe siècles), Paris, P.U.R., 2004, p. 113-149.

19. L. Benoit à la Guillaume, «Stylistique de l'éloge du héros ordinaire dans les discours sur l'état de l'Union », dans Études de stylistique anglaise, 10 (« Confluences »), 2017, p. 15-28.

20. B. Cassin, op. cit., p. 202.

21. K. Hall Jamieson, Eloquence in an Electronic Age. The Transformation of Political Speechmaking, Oxford University Press, 1988.

22. Ibid., p. 84.

23. L. Benoit à la Guillaume, Quand la Maison-Blanche prend la parole, op. cit., p. 2 ; et L. Cannon, President Reagan. The Role of a Lifetime, New York, Simon and Schuster, 1991, p. 99-100.

24. P. Noonan, What I Saw at the Revolution. A Political Life in the Reagan Era, New York, Random House, 1990, p. 82.

25. "These are the boys of Pointe du Hoc. These are the men who took the cliffs. These are the champions who helped free a continent. These are the heroes who helped end a war. »Cité et traduit par L. Benoit à la Guillaume, Quand la Maison-Blanche prend la parole, op. cit., p. 87. 
26. K. K. Campbell et K. H. Jamieson, Deeds Done in Words: Presidential Rhetoric and the Genres of Governance, Chicago, The University of Chicago Press, 1990, p. 70 et 73.

27. L. Benoit à la Guillaume, «Les stratégies du sortant lors des élections présidentielles américaines », Mots, les langages du politique, 112, Lyon, ENS Editions, 2016, p. 117-124.

28. Ibid.

29. L. W. Bennett, News : The Politics of Illusion, New York, Longman, 1988, p. 23-63.

30. G. C. Edwards III, On Deaf Ears: The Limits of the Bully Pulpit, New Haven, Yale University Press, 2003.

\section{AUTEUR}

\section{LUC BENOIT À LA GUILLAUME}

Université de Rouen, ERIAC 